\title{
Lifestyle Factors Related to Mortality and Survival: A Mini-Review
}

\author{
D. Rizzuto ${ }^{a} \quad$ L. Fratiglioni $i^{a} b$ \\ ${ }^{a}$ Aging Research Center (ARC), Department of Neurobiology, Care Sciences and Society, Karolinska Institutet and

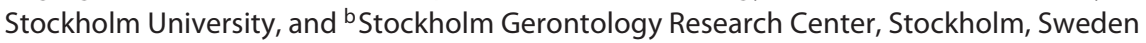

\section{Key Words}

Life habits · Social network · Leisure activity .

Elderly people $\cdot$ Mortality $\cdot$ Survival

\begin{abstract}
As the world's population ages, elderly people are becoming an increasingly important group that merits special attention with regard to health and social issues. Lifestyles affect health and survival at all ages, but the consequences of poor lifestyle behaviors may be different for elderly people than for younger adults. They can also be heavily dependent on exposure earlier in life. Our current state of knowledge is based predominantly on studies conducted among middleaged adults or young elderly people. Moreover, studies are sparse throughout the entire older age spectrum, from 65 to 90 years. This article summarizes the evidence regarding the impact of lifestyle behaviors on mortality among elderly people. It focuses on behaviors modifiable by individual actions and public health interventions, such as smoking, obesity and sedentary behavior, which predispose numerous people to diseases that rank among the leading causes of death, including heart disease, cancer, stroke, diabetes and dementia. These factors not only shorten life but, when they occur together, also have a major impact on survival beyond that associated with each single lifestyle factor. We propose an integrated life course model to guide research on longev-
\end{abstract}

ity to answer questions that remain open and to find new strategies to ensure a longer and healthier life for future generations.

(c) 2014 S. Karger AG, Basel

\section{Introduction}

Over the last 50 years, improvements in standards of living (income, nutrition and education) and health care (curative and preventive medicine) have resulted in a significant increase in life expectancy for adults of all ages, including those of advanced age [1]. Evidence from several studies indicates that longevity results from the combined effect of a variety of underlying factors, including genetic, environmental and medical factors, and that there is also a stochastic component [1]. Of these elements, modifiable risk factors (such as lifestyle factors) are especially relevant as they are amenable to intervention.

People who live in Sardinia (Italy), Okinawa (Japan) and Loma Linda (Calif., USA) have a significantly longer life expectancy than people in other parts of the developed world. A study conducted in these areas found that these populations have common lifestyle characteristics that may explain their longevity. These include: abstaining from smoking; constant and moderate physical activ- 
ity; social engagement; and a diet rich in vegetables, fruits and whole grains [2]. Indeed, according to the World Health Organization, lifestyle factors lie at the root of noncommunicable diseases such as cancer and cardiovascular diseases [3]. Dementia is also strongly associated with lifestyle factors [4]. Among people 75 years and older, cancer shortens life by approximately 4.5 years, and people with cardiovascular diseases and dementia live 3.5 years less than expected [5]. Further, dementia is the strongest determinant of functional dependence and institutionalization [5].

Identification of the possible determinants of human longevity is still an ongoing effort, and the underlying social and biological pathways remain to be clarified. However, it is clear that none of the factors identified to date is necessary or sufficient to determine the aging phenotype at the individual level, whereas it is likely that people may achieve exceptional longevity in multiple ways by engaging in different combinations of protective behaviors. This review aims to summarize the evidence concerning the effect(s) of key health behaviors on mortality and survival among older adults living in the more developed countries. We will limit our review to the most common modifiable lifestyle factors, including smoking, alcohol consumption, body weight, social network, physical activity and leisure time activity. Finally, we will discuss an integrative model that explores longevity and health in aging from a life course perspective to better address those questions that remain to be answered.

\section{Smoking}

Smoking is a global public-health concern. Worldwide, smoking causes more than 5 million deaths per year, and current trends show that it will cause more than 8 million deaths annually by 2030 [6]. The use of tobacco is a risk factor for at least 5 of the 10 leading causes of death worldwide: ischemic heart disease; cerebrovascular disease; tracheal, bronchial and lung cancers; lower respiratory infections; and chronic obstructive pulmonary disease [7]. Although most studies on these topics primarily focused on middle-aged adults, epidemiological evidence shows that smoking remains a strong risk factor for premature mortality even in old age. Even though the deleterious effects remain after quitting, risks decline over time. Smoking cessation is therefore beneficial at any age [8].

A meta-analysis of 17 studies on the impact of smoking on all-cause mortality in people $\geq 60$ years found that mortality was 83 and $34 \%$ higher in current and former smokers, respectively, than in never-smokers. Relative mortality of current and former smokers decreased slightly with increasing age [8]. In addition, this meta-analysis demonstrated that the relative mortality risk of former smokers decreased with time since smoking cessation, even when cessation occurred at older age [8]. Moreover, it has been estimated that smokers who survived to 75 years had a 1-year-shorter median survival than those who had never smoked [9]. In contrast, studies among nonagenarians [10] and centenarians [11] have not found a significant association between current and/or past smoking habits and longevity.

\section{Possible Mechanisms}

One possible explanation for the decrease in relative mortality with increasing age is the 'depletion of susceptibles' effect [8]. Smokers who are still alive at older age may have a genetic background or environmental characteristics that protect them from the harmful effects of tobacco smoking, whereas smokers who were more susceptible died at younger ages. A second explanation for the decrease in relative mortality at older ages may be the steep rise in absolute mortality above 70 years in both smokers and nonsmokers, which would attenuate the magnitude of the relative effect [8].

\section{Alcohol Consumption}

Excessive alcohol consumption is one of the leading preventable risk factors for death worldwide [7]. In his seminal work from 1926, Raymond Perl reported both a beneficial effect of light-to-moderate drinking and a detrimental effect of heavy drinking [12]. Further detail is revealed when mortality risk is examined by age group. The J-shaped association between alcohol intake and mortality has been established almost exclusively in studies of younger and middle-aged adult populations. In older people, however, this association has not been confirmed [13].

In a systematic review, Reid et al. [13] summarized existing evidence regarding the health-related effect of alcohol consumption on adults $\geq 60$ years. Among the 21 studies examined, 4 found that those who consumed between 14 and 19 drinks a week had a higher risk of mortality than nondrinkers. In contrast, 4 studies that used a comparable definition of alcohol consumption reported an inverse association between mortality and increased alcohol intake. Furthermore, another 13 studies found no association between alcohol intake and mortality among people $\geq 60$ years [13]. 


\section{Possible Mechanisms}

A high level of alcohol consumption has been found to be associated with a higher risk of hypertension, alcoholic cardiomyopathy, cancer, cerebrovascular events and dementia [14]. On the other hand, several mechanism have been proposed to explain the health benefits on the heart of moderate alcohol consumption, such as an increase in high-density lipoprotein cholesterol, a reduction in plasma viscosity and fibrinogen concentration, an increase in fibrinolysis, a decrease in platelet aggregation, improvement in endothelial function, a reduction in inflammation and the promotion of antioxidant effects [14]. Even so, the evidence regarding the possible health benefits of alcohol is uncertain, and alcohol may not benefit everyone who drinks.

Possible explanations for the inconsistent findings from previous studies are the variation in the study-specific cutoff points for alcohol intake, the choice of reference group, and likely classification errors. Underreporting of alcohol use could also have prevented the detection of adverse effects. Finally, the 'survival cohort effect' should also be taken into account: people with alcohol-related diseases could die prematurely, leaving a cohort which is less susceptible to the adverse effects of alcohol [13]. However, many biological changes linked to aging could make older adults more vulnerable to excessive alcohol consumption. Older adults are more likely than younger adults to have multiple comorbidities [15] and to use more medication [13]. Alcohol may interact differently with different chronic diseases and with medication to adversely affect health outcomes. Thus, caution is necessary in the interpretation of the inverse association between alcohol intake and mortality in elderly people.

\section{Body Weight}

The body mass index (BMI) is a reasonably good measure of general adiposity, and a high BMI is an established risk factor for several causes of death, including: ischemic heart disease; stroke; and cancer of the large intestine, kidney and endometrium [16]. In a collaborative analysis of 57 prospective studies that included almost 900,000 adults, overall mortality was lowest in those of any age whose BMI was between 22.5 and 25. Above this range, each 5-point increase in BMI was associated with about $30 \%$ higher mortality. Although the proportional increase was greater at younger ages (35-59 years), the corresponding increase in mortality for those in their 70 s was

Lifestyle Factors Related to Mortality and Survival: A Mini-Review nearly $30 \%$, and for those in their 80 s, it was $15 \%$ [16]. This finding suggests that the harmful effects of overweight and obesity on survival are weaker in elderly people than in younger adults. Indeed, a review of 13 prospective studies of people aged $\geq 65$ years (followed up for between 3 and 23 years), found that mild-to-moderate overweight (BMI range: $20-27$ ) did not seem to be a risk factor for all-cause mortality. In addition, in the few studies that found a significant association between BMI over 27 and mortality, the association seems to disappear among individuals $\geq 75$ years [17]. The same conclusions were drawn in another meta-analysis, which included 32 studies: BMI in the overweight range (25-29.9) was not associated with increased mortality, and BMI in the obese range (30-34.9) was associated with a modest increase (about 10\%) [18]. In addition, a number of studies have reported increased mortality among elderly people in the lowest BMI categories [16]. The mortality rate tends to increase when the BMI falls below 20 .

\section{Possible Mechanisms}

Several hypotheses have been proposed to explain the weak association between high BMI and mortality in elderly people [18]. First, it is possible that, as with smoking and alcohol consumption, those most susceptible to the effects of the risk factor die when they are younger, leaving a resistant older population. Second, it has been found that the relation between BMI, body fat and fat distribution is weaker in older adults than in younger adults. Since the impact of high BMI on mortality is explained by the increase in total and abdominal fat mass, it is not surprising that a quicker association of abdominal fat deposition with BMI in older adults leads to a weaker effect of high BMI on mortality in elderly than in younger people. Finally, BMI is a reflection of lean mass. There is an inverse association between lean mass and mortality risk, which is thought to reflect the fact that lean mass acts as a nutritional reserve during prolonged periods of illness and disease. A high lean mass in elderly people with a high BMI may offer some protection against mortality.

Being underweight was associated with loss of peripheral and respiratory muscle mass [19], which might increase vulnerability to acute infectious diseases [20]. Moreover, lower BMI may be linked to existing illnesses or conditions that are the real cause of the increased mortality. The relationship between BMI and mortality in older adults may be confounded by several covariates. One such covariate is weight loss, especially unintentional weight loss, which is generally associated with chronic 
diseases, such as dementia [21], and with mortality [18]. Another important potential confounder that should be taken into account is smoking; smokers are thinner and have higher mortality than nonsmokers [18, 22].

Long-term follow-up is needed to clarify the effects of BMI on health in elderly people. Obesity-related consequences take years to develop; thus, elderly people who become obese in old age may die of other conditions unrelated to obesity before they develop obesityrelated health problems. This may be why studies with short follow-up periods generally show associations between low but not high BMI and mortality, whereas studies with longer follow-up times show significant associations between high BMI and mortality in elderly people [22].

\section{Social Network}

The influence of social relations on mortality is well documented. A meta-analysis of 148 studies $(308,849$ participants followed for an average of 7.5 years) confirms that social network significantly predicts mortality [23]. People with social relationships defined as adequate are $50 \%$ more likely to survive than older adults with social relationships defined as poor or insufficient. The overall effect remains consistent across age [23]. However, it is unclear whether specific types of relationships are more advantageous than others. Giles et al. [24] showed that the beneficial association between social networks and survival among elderly people may be restricted to relationships with friends and confidants rather than with children and relatives. In a meta-analysis of 53 prospective observational studies on older adults, marriage or support from a partner was found be a significant independent predictor of survival; the overall reduction in mortality risk was $9-15 \%$. However, this association was statistically significant in only half of the studies [25].

\section{Possible Mechanisms}

One hypothesis suggests that strong social connections can buffer significant stress and protect against negative stress-related outcomes. People might be protected from adopting stress-related lifestyle choices that are detrimental to health, such as smoking, excessive alcohol consumption and sleep loss [25]. The main-effect hypothesis postulates that social support is beneficial regardless of the stress level a person is experiencing; in other words, social support can act protectively even before a stressor is experienced. For instance, persons with social support could be influenced to engage in protective behaviors such as exercise. Moreover, having a wide range of social ties also provides multiple sources of information that could result in more effective use of available health care and services [26].

\section{Physical and Leisure Time Activity}

The majority of the studies that investigate the relationship between physical and leisure activities and health focus mainly on younger or middle-aged populations [27]. Although it is still unclear whether these results are completely generalizable to older people, a growing body of empirical evidence suggests that being active and participating in different kinds of leisure activities also has a positive effect on survival in adults of advanced age.

\section{Physical Activity}

A study of approximately 13,000 elderly people (median age at baseline: 74 years) followed up for 28 years showed that those who spent any amount of time in physical activity (even 30 min per day) had a $15-35 \%$ lower risk of mortality than those who spent no time in physical activity [28]. A population-based cohort study on 2,357 men aged $\geq 65$ years found that those who exercised rigorously enough to cause sweating 2-4 times per week were $30 \%$ less likely to die than those who exercised 1 or fewer times per week [29]. Similar results have been found for the 2,600 participants aged $\geq 75$ years enrolled in the SENECA (Survey in Europe on Nutrition and the Elderly, a Concerted Action) [30] and for participants in the Italian Silver Network Home Care Project (2,244 people aged $\geq 70$ years who were followed up for at least 1 year) [31]. In a study on 1,810 Swedish men and women aged $\geq 75$ years, researchers found that those who were physically active survived 2 years longer that those who were physically inactive [9]. Comparison between these studies is not easy because various definitions of physical activity and activity levels were used.

\section{Leisure Time Activity}

Far fewer studies have analyzed the association between activities that have also a physical component and mortality in elderly adults. In a study on 2,761 American men and women aged $\geq 65$ years, researchers found that higher participation in social activities (such as attending church, going to the movies, to the theater or to sporting 
events, and playing games) decreased mortality by $19 \%$, and higher participation in productive activities (such as gardening, shopping, community work and cooking) decreased it by $23 \%$ [32]. In a Swedish cohort of 463 people aged $\geq 77$ years, greater participation in solitary activities with a physical component (gardening and other hobbies) was associated with a $20 \%$ reduction in mortality [33]. In a 6-year follow-up analysis of 2,291 people aged 67-95 years, a greater overall activity level was associated with reduced mortality. Activities were classified as follows: social activities (visiting family and friends, church-related activities and sports), and productive activities (volunteer work, housework, gardening and yard work) [34]. In the Leisure World Cohort Study, 13,000 elderly men and women (median age: 74 years) were followed up for 28 years, and participation in less physically demanding activities reduced mortality by $15-30 \%$ [28]. Results from a Swedish cohort of 1,246 people (mean age: 75 years), followed up for 12 years, showed that those who participated in 2 or fewer activities had twice the mortality risk as those who participated in 6 or more activities [35].

\section{Possible Mechanisms}

There are several putative mechanisms whereby physical and leisure time activities may reduce the risk of mortality. Both physiological and psychosocial pathways may be involved, perhaps simultaneously [35]. For instance, physical activity is associated with beneficial changes in the metabolic profile, including a decrease in body weight and in blood concentrations of insulin, proinsulin, glucose and lipids. An improved metabolic profile reduces the prevalence of the metabolic syndrome, obesity and cardiovascular disease [36]. Other suggested mechanisms for the effect of physical activity on mortality include reduced mortality due to cancer. Reductions in cancer mortality may be related to reduced fat stores, increased energy expenditure, changes in sex hormone levels, improved immune function, reductions in levels of insulin and insulin-like growth factors, and reduced generation of free radicals [36]. Specifically in older people, physical activity is also important for maintaining muscle strength, postural balance, functional independence and healthrelated quality of life [36]. All these factors protect against falls as well as against depression and other mental disorders. Psychosocial pathways also link physical activity to survival because participation in physical activity among elderly people is often related to the presence of an exercise partner such as a spouse or friend [35].

Leisure time activity seems to affect survival primarily through psychosocial pathways. Social participation of-

Lifestyle Factors Related to Mortality and

Survival: A Mini-Review ten provides individuals with meaningful roles, greater social networks, and social support, all of which protect against damaging physiological responses by improving coping abilities and health behaviors [35].

\section{Combination of Health Behaviors}

Most studies have investigated the association between single lifestyle factors and health, although it seems that individual risk of mortality can only be minimized by implementing a combination of healthy behaviors. In the last decade, several large cohort studies have investigated the combined effects of lifestyle factors on various health outcomes, including mortality. However, few of the studies were carried out among elderly people.

In the study Healthy Ageing, a Longitudinal Study in Europe, 1,507 men and 832 women aged $70-90$ years were followed up for an average of 10 years. The researchers investigated 4 behaviors in relation to mortality: healthy diet, alcohol intake of $>0$ g per day, nonsmoking and adequate physical activity [37]. To assess the association, a lifestyle score was calculated by adding individual scores for all 4 behaviors. Diet was assessed using a modified version of the Mediterranean diet. The low-risk group was defined as those who had a score of $\geq 4$. The low-risk group for alcohol was defined as those who consumed $>0 \mathrm{~g}$ of alcohol per day. The low-risk group for smoking consisted of those who had never smoked or had stopped smoking $>15$ years ago. Individuals with a score in the intermediate and the highest tertile on the Voorrips or Morris questionnaire were considered the low-risk group for physical activity [37]. Those with low-risk scores for all 4 factors had $65 \%$ lower all-cause mortality than those with no or only 1 lowrisk behavior [37].

Similar results were also found in the collaborative European project SENECA, in which 631 men and 650 women aged 70-75 years were followed up for 10 years [38]. Researchers found that those with 3 unhealthy lifestyle factors had a 3- to 4 -fold increased mortality risk. The lifestyle score ranged from 0 (poor) to 3 (good). It was calculated by adding the scores for 3 lifestyle factors: (1) physical activity (lowest tertile of activity vs. the intermediate and highest tertiles); (2) diet as measured by the Mediterranean diet score (low-quality diet group with a score of $\leq 4$ vs. the high-quality diet group with a score of $>4$ ); and (3) smoking (current smokers and those who quit $\leq 15$ years before vs. those who had never smoked or had quit $>15$ years before) [38]. 


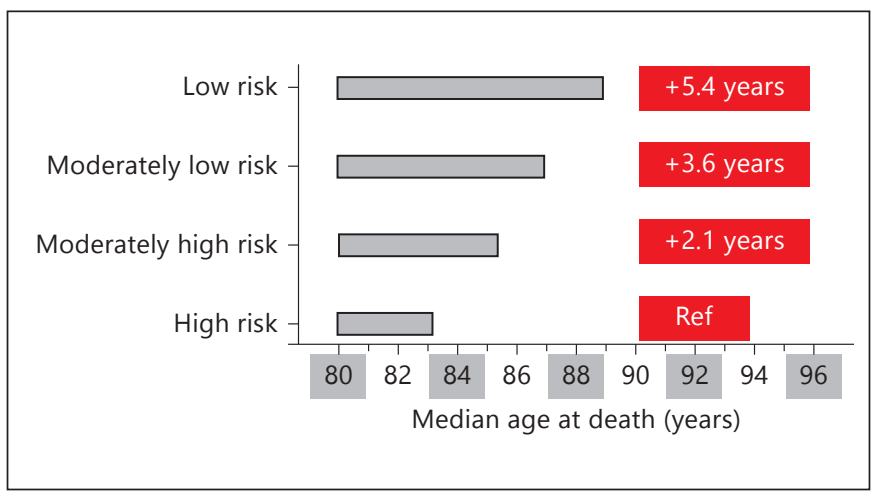

Fig. 1. Differences in median age at death in 4 risk-profile groups after 18 years of follow-up. High-risk profile: unhealthy life habits (over- or underweight and current or former smoker), no participation in leisure activities, and a limited or poor social network; moderate- to high-risk profile: at least 2 of the 3 risk factors; moderate- to low-risk profile: only 1 of the 3 risk factors; low-risk profile: no risk factors.

An Australian study showed that healthy lifestyle score was inversely related to mortality among 7,989 men aged 65-83 years, followed up for 5 years [39]. The score indicated how many of 8 behaviors each individual followed: (1) lifelong nonsmoker or nonsmoker for $\geq 1$ year; (2) engaged in physical activity $\geq 3 \mathrm{~h}$ weekly; (3) drank $\leq 20$ g of alcohol daily; (4) ate fish $\geq 3$ times weekly; (5) ate meat $<5$ times weekly; (6) never added salt to food; (7) had a selfreported BMI of $\leq 25.0$; and (8) used reduced-fat or skim milk.

In the Kungsholmen Project, a Swedish cohort of 1,810 participants $\geq 75$ years old followed up for 18 years, a risk profile was defined based on 3 unhealthy behaviors. Individuals with the lowest risk profile lived more than 5 years longer than those with the highest risk profile (fig. 1). These associations, although attenuated, were still present among the oldest old (those $\geq 85$ years of age) and those with chronic diseases [9]. Participants with the lowest risk profile had a BMI of 20-25, had never smoked, had a rich social network, and participated in leisure activities each month. Participants were considered to have a rich social network if they met at least 2 of the 3 following criteria: (1) being married or living with someone; (2) having children with whom they were in daily-to-weekly contact, and finding this level of contact satisfactory; and (3) having relatives or friends with whom they were in daily-to-weekly contact, and finding this level of contact satisfactory. Participants were considered to be active in leisure activities if they took part in mental, physical, social or productive activity [9].
A potential weakness of all the studies described here is the use of simple tallies of healthy or unhealthy behaviors to create health behavior scores; neither the possibly different weights of each factor nor their potentially multiplicative effects were taken into account. On the other hand, a simple score is easier to understand and use in clinical practice than a complicated algorithm, and a great deal of the importance of health behavior scores lies in their potential usefulness and effectiveness in the field of clinical health promotion.

\section{Methodological Issues}

A number of methodological issues must be considered when studying elderly people. First, surveys of older people include high proportions of dropouts (largely due to mortality) and high proportions of nonresponders. Selective survival bias is a concern in cohort studies in which survival is somehow related to the probability of the studied outcome. In studies of lifestyle and mortality, those who die or refuse to participate before baseline are most likely sicker or engage in less healthy lifestyle behaviors than participants. Thus, it is likely that those dropouts lead to an underestimation of the association between the exposure and outcome. Attrition of older respondents may also be an issue in longitudinal studies and has essentially the same effect as refusals to participate at baseline. A variety of strategies can be adopted to compensate for bias introduced by nonresponse at baseline and follow-up. For instance, people who could not be contacted, who refused to participate, or who died can be replaced by others with similar characteristics. Another strategy is the use of proxy respondents, which can compensate for attrition due to incapacitation. However, the use of proxy respondents may introduce the potential for systematic under- or overreporting.

Second, the aging process is associated with physiological changes such as decline in cognitive function. Cognitive function may affect not only the understanding of survey questions and instructions, but also the respondent's ability to provide accurate information.

Third, validity in the assessment of an exposure is especially relevant when studying lifestyle factors in older people. Risk factor levels obtained at older age may not necessarily reflect characteristic values over the preceding year, because current values may have been influenced by changes in underlying function, undetected disease or social processes. Nevertheless, even in those people with known diseases, subsequent survival is strongly related to 
health behaviors. Moreover, when the exposures of interest are based on self-reported data, some degree of misclassification is inevitable. Older adults may be resistant to reporting potentially embarrassing information and inclined to overreport desirable behaviors. However, if the exposure information is collected before the occurrence of the outcome, the classification errors are independent of the future outcome; therefore it would most likely attenuate the observed findings.

Fourth, reverse causality is an important issue in the study of physical and leisure time activity. For instance, poor health may not be the reason for but rather the cause of inactivity. Healthier individuals tend to participate in leisure activity to a greater extent than those who are less healthy, and they may also be inclined to participate more regularly and with greater intensity [35]. Illness is thought to play a key role in molding our social network too. Illness may result in poorer or more restricted social relationships, such that individuals closer to death may have less social support than healthy individuals [23]. To limit bias from reverse causality, researchers might perform subgroup analyses of the healthiest participants.

Finally, it is also possible that personality traits (e.g. extroversion, attachment and hostility) account for associations between social network and health. A number of traits that are thought to influence one's social relationships have been implicated in health outcomes. Hostile people, for example, are at greater risk of coronary artery disease and possibly other physical health problems [26]. Further studies are necessary to better clarify these pathways.

\section{Conclusion}

This study set out to summarize evidence about the association between the most common modifiable lifestyle factors and survival in advanced age. The evidence suggests that the benefits of healthy lifestyle behavior and social support are even seen in older adults. Living a healthier lifestyle, even when older, leads to better physical function, may reduce susceptibility to disease, and may be one of the keys to longevity.

From a public-health perspective, the ability to identify a group of individuals at risk of shorter and unhealthier lives may lead to primary and secondary preventive interventions. Interventions that promote a healthy lifestyle in older adults would have a profound impact on both individuals and society, reducing suffering and carerelated costs.

Lifestyle Factors Related to Mortality and Survival: A Mini-Review

\section{Open Questions and Future Perspectives}

In the last decade, it has become more and more evident that most of the important age-related health outcomes (including mortality, cardiovascular diseases and mental disorders) are interrelated and are likely associated with similar risk factors. To better understand the mechanisms behind these conditions, we should consider the complex interplay among demographic factors, genetic susceptibility, biological factors and environmental exposure, and how their interactions affect various health outcomes, including survival. Combinations of beneficial individual lifestyle factors may have a synergistic protective effect against different lethal diseases, which could explain the reduced mortality rates associated with combinations of healthy lifestyle factors. Moreover, biological, social and psychological factors may not contribute equally to health in aging. It is also likely that the probability of survival at advanced age is not determined in any single period of the lifespan.

The challenge now is to understand the interplay among these multiple factors as such an understanding may facilitate the identification of those who are more prone to experience adverse health-related conditions in late life, and consequently functional impairment, disability and premature death. We propose the use of an integrative approach in which factors that lead to healthier aging are explored from a life course perspective (fig. 2). The purpose of the life course approach is to build and test theoretical models that have the potential to show pathways linking exposure across the life course to late-life health outcomes [40]. This approach will help identify the windows in time when exposure has its greatest effects on later outcomes. It will also permit us to determine whether accumulated exposure could have multiplicative or additive effects over the life course.

Older adults are a highly heterogeneous group. People age at different rates and range widely in their vulnerability and exposure to risk factors, and therefore in morbidity and mortality. Our model takes into account many of the variables active during different life periods (childhood, youth, middle age, transitional phase $/ 60-74$ years, and old age) that render the older population so heterogeneous. These include: genetic background; a broad range of social, environmental and biological factors; and medical conditions (fig. 2). We consider health as a multidimensional condition and its changes as a continuous process that begins with the development of symptoms related to underlying biological changes and 
Fig. 2. An integrated life course model to explain health and survival to old age. Health is described as a multidimensional outcome.

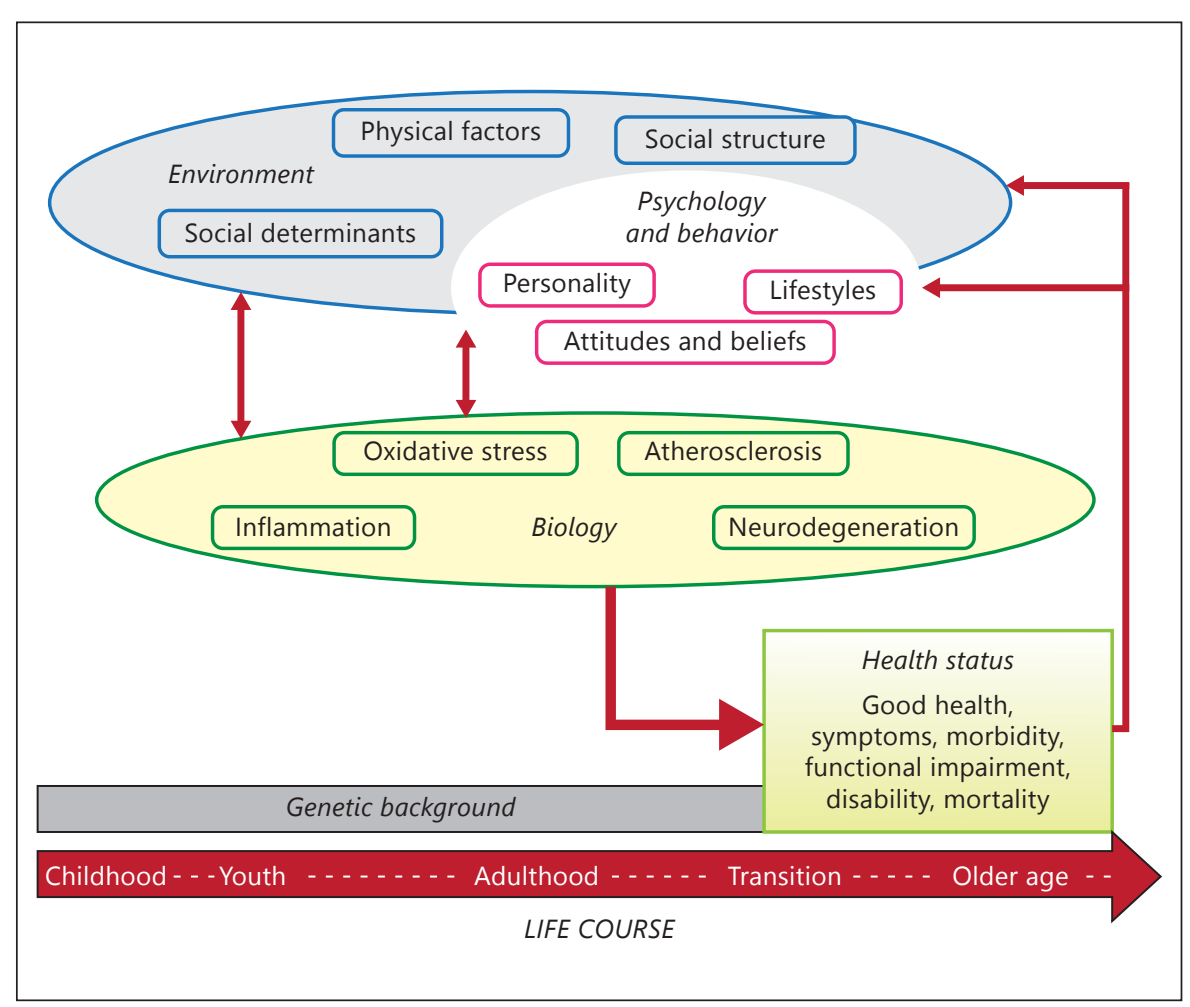

continues to disease onset, functional loss and disability, and ultimately terminal status and death. Our model identifies several potential interacting pathways to explain different health outcomes, which include lifetime environmental, psychological, behavioral and biological conditions. Environmental influences act at different levels, from social structure (income distribution in society, gender equality in society) and physical components (pollution, indoor environments) to the individual level (socioeconomic status, education). Behavioral influences play a role, as do psychological influences, which include factors such as personality, attitudes and beliefs. Many dietary and lifestyle factors are important in the etiology of noncommunicable diseases. Such factors include - but are not limited to - food, nutrients, energy intake, physical activity, obesity, smoking, alcohol consumption, behavioral factors (e.g. sleep, stress) and cultural factors. Although these environmental, psychological and behavioral factors contribute to health status in different ways, they all affect health outcomes via biological mechanisms such as cardiovascular lesions, oxidative stress, neurodegeneration and inflammation. In addition to all these factors, which are active over the lifetime, an individual's genetic composition also plays a role in longevity. This effect is likely the re- sult of multiple genes, each of which makes a modest contribution.

The integrative approach proposed in the model will help us answer open questions, such as: How detrimental are discontinuous healthy behaviors to longevity? Can people still experience beneficial effects on longevity if they introduce behavioral changes at advanced ages? Is it true that it is never too late to adopt healthy behaviors and/or eliminate unhealthy ones? How much do psychological experiences influence health behaviors? How different are health behaviors in different generations? Can we predict any trends for the next 50 years? The availability of large cohort studies that include people of middle age and older will give us answers to these questions in the near future.

\section{Acknowledgments}

This study was supported by grants from the Swedish Council for Working Life and Social Research, the Swedish Research Council for Medicine, Swedish Brain Power, Stiftelsen Ragnhild och Einar Lundströms Minne, Gun och Bertil Stohnes Stiftelsen, Stiftelsen för Gamla Tjänarinnor and Stiftelsen Solstickan. We thank Sara Angleman and Kimberly Kane (scientific editors) for useful comments on the text. 


\section{References}

1 Christensen K, Doblhammer G, Rau R, Vaupel JW: Ageing populations: the challenges ahead. Lancet 2009;374:1196-1208.

2 Buettner D: The secrets of living longer. $\mathrm{Na}$ tional Geographic, November 2005.

3 World Health Organization: Global status report on noncommunicable diseases 2010. Geneva, World Health Organization, 2011.

4 Fratiglioni L, Paillard-Borg S, Winblad B: An active and socially integrated lifestyle in late life might protect against dementia. Lancet Neurol 2004;3:343-353.

$\checkmark 5$ Rizzuto D, Bellocco R, Kivipelto M, Clerici F, Wimo A, Fratiglioni L: Dementia after age 75: survival in different severity stages and years of life lost. Curr Alzheimer Res 2012;9:795-800.

6 World Health Organization: WHO report on the global tobacco epidemic, 2011: warning about the dangers of tobacco. Geneva, World Health Organization, 2011.

$>7$ Murray CJ, Lopez AD: Measuring the global burden of disease. N Engl J Med 2013;369: 448-457.

8 Gellert C, Schöttker B, Brenner H: Smoking and all-cause mortality in older people: systematic review and meta-analysis. Arch Intern Med 2012;172:837-844.

$>9$ Rizzuto D, Orsini N, Qiu C, Wang HX, Fratiglioni L: Lifestyle, social factors, and survival after age 75: population based study. BMJ 2012;345:e5568.

-10 Rajpathak SN, Liu Y, Ben-David O, Reddy S, Atzmon G, Crandall J, Barzilai N: Lifestyle factors of people with exceptional longevity. J Am Geriatr Soc 2011;59:1509-1512.

$>11$ Hagberg B, Samuelsson G: Survival after 100 years of age: a multivariate model of exceptional survival in Swedish centenarians. J Gerontol A Biol Sci Med Sci 2008;63:1219-1226.

12 Pearl R: Alcohol and Longevity. New York, Knopf, 1926.

$>13$ Reid MC, Boutros NN, O’Connor PG, Cadariu A, Concato J: The health-related effects of alcohol use in older persons: a systematic review. Subst Abus 2002;23:149-164.

$>14$ Kloner RA, Rezkalla SH: To drink or not to drink? That is the question. Circulation 2007; 116:1306-1317.

-15 Marengoni A, Angleman S, Melis R, Mangialasche F, Karp A, Garmen A, Meinow B, Fratiglioni L: Aging with multimorbidity: a systematic review of the literature. Ageing Res Rev 2011;10:430-439.
16 Whitlock G, Lewington S, Sherliker P, Clarke R, Emberson J, Halsey J, Qizilbash N, Collins $\mathrm{R}$, Peto R: Body-mass index and cause-specific mortality in 900,000 adults: collaborative analyses of 57 prospective studies. Lancet 2009;373:1083-1096.

17 Heiat A, Vaccarino V, Krumholz HM: An evidence-based assessment of federal guidelines for overweight and obesity as they apply to elderly persons. Arch Intern Med 2001;161: 1194-1203.

$>18$ Janssen I, Mark AE: Elevated body mass index and mortality risk in the elderly. Obes Rev 2007;8:41-59.

19 Engelen MP, Schols AM, Baken WC, Wesseling GJ, Wouters EF: Nutritional depletion in relation to respiratory and peripheral skeletal muscle function in out-patients with COPD. Eur Respir J 1994;7:1793-1797.

20 Potter JF, Schafer DF, Bohi RL: In-hospital mortality as a function of body mass index: an age-dependent variable. J Gerontol 1988; 43:M59-M63.

21 Atti AR, Palmer K, Volpato S, Winblad B, de Ronchi D, Fratiglioni L: Late-life body mass index and dementia incidence: nine-year follow-up data from the Kungsholmen Project. J Am Geriatr Soc 2008;56:111-116.

22 Zamboni M, Mazzali G, Zoico E, Harris TB, Meigs JB, di Francesco V, Fantin F, Bissoli L, Bosello O: Health consequences of obesity in the elderly: a review of four unresolved questions. Int J Obes (Lond) 2005;29:1011-1029.

23 Holt-Lunstad J, Smith TB, Layton JB: Social relationships and mortality risk: a meta-analytic review. PLoS Med 2010;7:e1000316.

24 Giles LC, Glonek GF, Luszcz MA, Andrews GR: Effect of social networks on 10 year survival in very old Australians: the Australian longitudinal study of aging. J Epidemiol Community Health 2005;59:574-579.

25 Manzoli L, Villari P, Pirone GM, Boccia A: Marital status and mortality in the elderly: a systematic review and meta-analysis. Soc Sci Med 2007;64:77-94.

26 Cohen S: Social relationships and health. Am Psychol 2004;59:676-684.

27 Woodcock J, Franco OH, Orsini N, Roberts I: Non-vigorous physical activity and all-cause mortality: systematic review and meta-analysis of cohort studies. Int J Epidemiol 2010;40 121-138.

28 Paganini-Hill A, Kawas CH, Corrada MM: Activities and mortality in the elderly: the Leisure World Cohort Study. J Gerontol A Biol Sci Med Sci 2011;66:559-567.

-29 Yates LB, Djoussé L, Kurth T, Buring JE, Gaziano JM: Exceptional longevity in men: modifiable factors associated with survival and function to age 90 years. Arch Intern Med 2008;168:284-290.
30 de Groot LC, Verheijden MW, de Henauw S, Schroll M, van Staveren WA: Lifestyle, nutritional status, health, and mortality in elderly people across Europe: a review of the longitudinal results of the SENECA study. J Gerontol A Biol Sci Med Sci 2004;59:1277-1284.

31 Landi F, Cesari M, Onder G, Lattanzio F, Gravina EM, Bernabei R: Physical activity and mortality in frail, community-living elderly patients. J Gerontol A Biol Sci Med Sci 2004; 59:833-837.

32 Glass TA, de Leon CM, Marottoli RA, Berkman LF: Population based study of social and productive activities as predictors of survival among elderly Americans. BMJ 1999;319: 478-483.

$>33$ Lennartsson C, Silverstein M: Does engagement with life enhance survival of elderly people in Sweden? The role of social and leisure activities. J Gerontol B Psychol Sci Soc Sci 2001;56:S335-S342.

34 Menec VH: The relation between everyday activities and successful aging: a 6-year longitudinal study. J Gerontol B Psychol Sci Soc Sci 2003;58:S74-S82.

35 Agahi N, Parker MG: Leisure activities and mortality: does gender matter? J Aging Health 2008;20:855-871.

36 Samitz G, Egger M, Zwahlen M: Domains of physical activity and all-cause mortality: systematic review and dose-response meta-analysis of cohort studies. Int J Epidemiol 2011;40: 1382-1400.

37 Knoops KT, de Groot LC, Kromhout D, Perrin AE, Moreiras-Varela O, Menotti A, van Staveren WA: Mediterranean diet, lifestyle factors, and 10-year mortality in elderly European men and women: the HALE project. JAMA 2004;292:1433-1439.

38 Haveman-Nies A, de Groot LP, Burema J, Cruz JA, Osler M, van Staveren WA: Dietary quality and lifestyle factors in relation to 10-year mortality in older Europeans: the SENECA study. Am J Epidemiol 2002;156: 962-968.

$>39$ Spencer CA, Jamrozik K, Norman PE, Lawrence-Brown M: A simple lifestyle score predicts survival in healthy elderly men. Prev Med 2005;40:712-717.

40 Ben-Shlomo Y, Kuh D: A life course approach to chronic disease epidemiology: conceptual models, empirical challenges and interdisciplinary perspectives. Int J Epidemiol 2002;31: 285-293 